\title{
Prevalence of vitamin D deficiency in Slovak women with polycystic ovary syndrome and its relation to metabolic and reproductive abnormalities
}

\section{Figurová J. ${ }^{1}$, Petríková J. ${ }^{1}$, Dravecká I. ${ }^{1}$, Javorský M. ${ }^{2}$, Lazúrová I. ${ }^{1}$}

11 st Department of Internal medicine, L. Pasteur University Hospital and Medical faculty of P.J. Šafárik University, Košice

$24^{\text {th }}$ Department of Internal medicine, L. Pasteur University Hospital and Medical faculty of P.J. Šafárik University, Košice

\section{OBJECTIVES}

Polycystic ovary syndrome (PCOS) is the most common endocrine disorder characterised by menstrual dysfunction, infertility, presence of polycystic ovaries and biochemical and clinical hyperandrogenism affecting up to $4-18 \%$ women of reproductive age [1]. There is an evidence that PCOS women suffer from various metabolic disturbances such as visceral obesity, insulin resistance, impaired glucose tolerance, dyslipidemia, type 2 diabetes mellitus and metabolic syndrome [2]. Vitamin D is thought to influence the development of PCOS through gene transcription and hormonal modulation of insulin metabolism and fertility regulation [3].

\section{METHODS}

- Study sample:

- 99 women with PCOS according to Rotterdam criteria

- 66 regularly menstruating controls

- Methods:

- 25(OH)D, anthropometric, endocrine and metabolic parameters were evaluated

- vitamin D deficiency: serum level of $25(\mathrm{OH}) \mathrm{D}$ $<30 \mathrm{ng} / \mathrm{ml}$

- insulin resistance: HOMA-IR $>2,5$

- metabolic syndrome according to NCEAP ATPIII

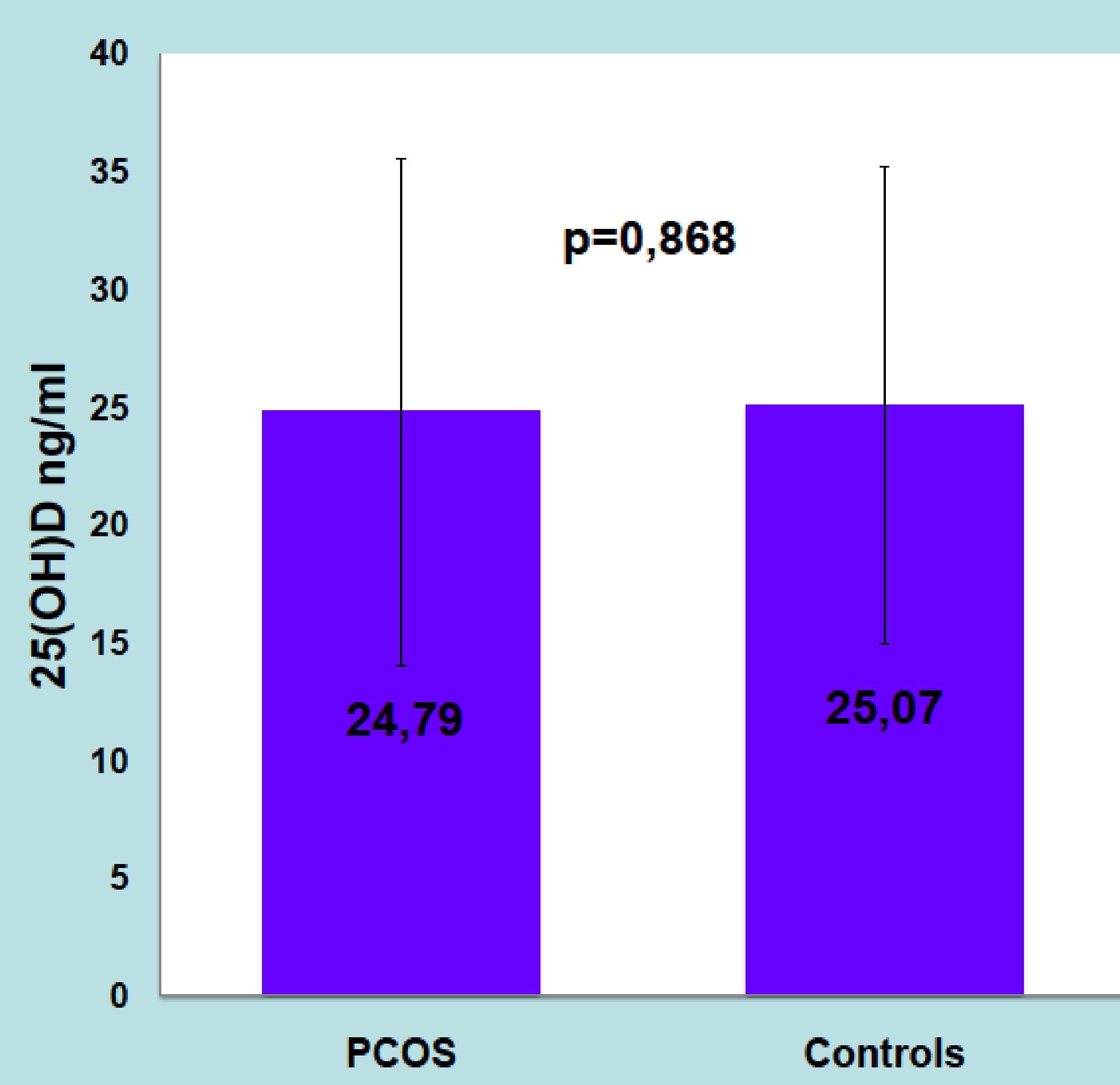

Figure 1. Serum levels of $25(\mathrm{OH}) \mathrm{D}$ in PCOS group and controls.

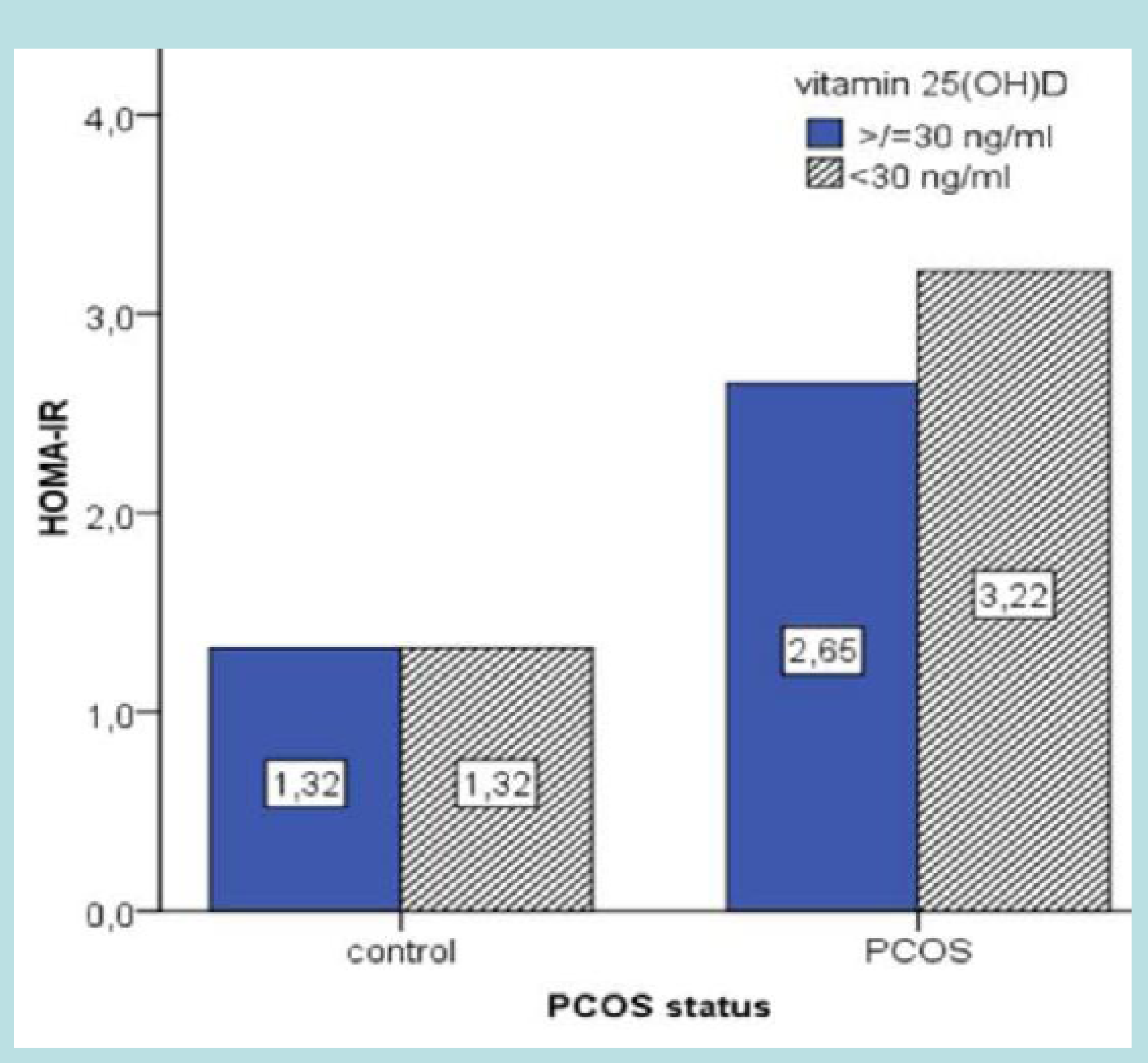

Figure 2. Comparison of HOMA-IR index between PCOS and control group in subgroup of vitamin $D$ deficient subjects $(\mathrm{p}<0.001$; adjusted for age, BMl: $\quad p$ adj $=0.036$ ) and vitamin $D$ non

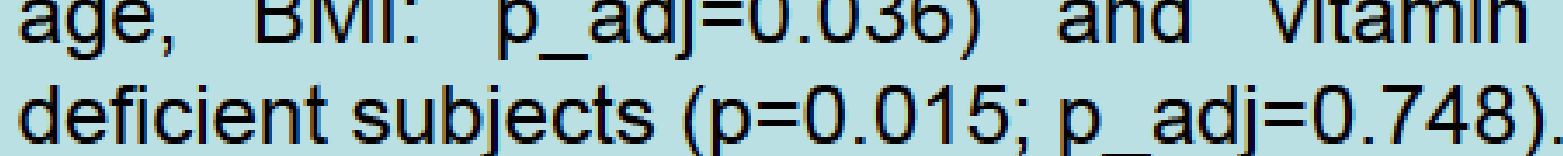
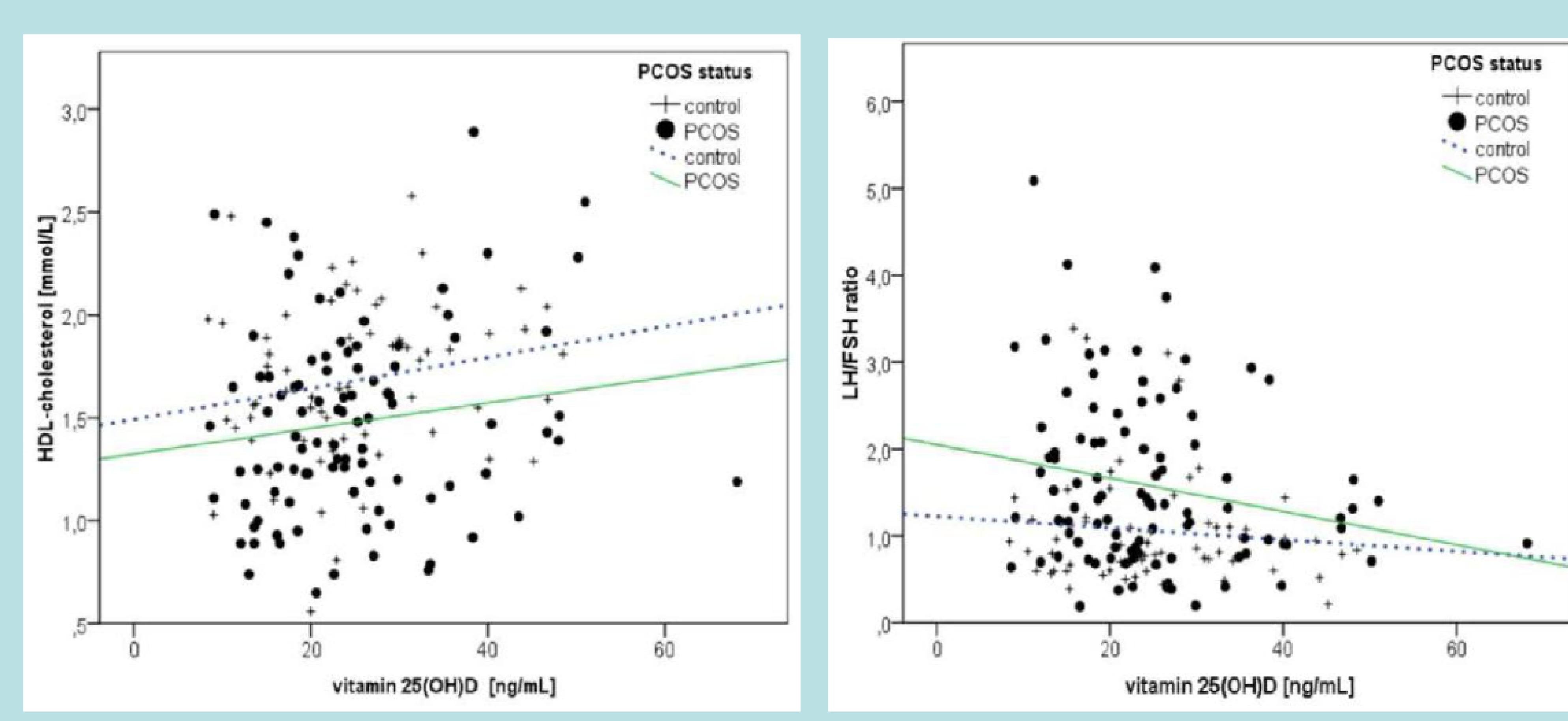

Figure 3. a) Positive correlation between $25(\mathrm{OH}) \mathrm{D}$ levels and serum HDL-cholesterol in all subjects $(r=0.159, p=0.043)$. b) Negative correlation between $25(\mathrm{OH}) \mathrm{D}$ levels and $\mathrm{LH} / \mathrm{FSH}$ ratio in PCOS $(r=-0.211, p=0.037)$.

\section{RESULTS}

There was no significant difference in $25(\mathrm{OH}) \mathrm{D}$ levels between PCOS women and controls $(24.79 \pm 10.77$ vs $25.07 \pm 10.14$ $\mathrm{ng} / \mathrm{ml}, \mathrm{p}=0.868$ ) and also in the prevalence of $25(\mathrm{OH}) \mathrm{D}$ deficiency in both groups ( 80 vs $70 \% ; \mathrm{p}=0.138)$. PCOS women with metabolic syndrome (MS) had lower serum $25(\mathrm{OH}) \mathrm{D}$ compared to those without MS $(20.6 \pm 8.3 \mathrm{vs} 25.9 \pm 11.3 \mathrm{ng} / \mathrm{ml}$, $p=0.049)$. PCOS women with vitamin $D$ deficiency had borderline higher serum triglycerides $(1.44 \pm 0.93$ vs $1.03 \pm 0.46$, $p=0.051$ ) and tended to have higher HOMA-IR (median [quartiles]: 2.24 [1,38- 3,51] vs 1,85 [1,04-3,68], $p=0.467$ ) compared to PCOS with sufficient vitamin D levels. 25(OH)D correlated positively with HDL-cholesterol in all subjects $\left(r=0.159, p=0.043\right.$; $p$ adjusted for age, $\left.B M I, p \_a d j=0.03\right)$ and negatively with $L H / F S H$ ratio $(r=-0.211, p=0.037)$ in $P C O S$.

\section{CONCLUSIONS}

We demonstrate high prevalence of vitamin $D$ deficiency in the population of young women in reproductive age with no difference in serum vitamin $D$ concentration between PCOS patients and control group subjects. Low vitamin D status was associated with presence of the metabolic syndrome in PCOS women. Insulin resistance and other metabolic and reproductive abnormalities in PCOS women seem to be related to PCOS rather than to vitamin $D$ deficiency. It was already described that both vitamin $D$ deficiency and PCOS are associated with features of metabolic syndrome, but it is still unclear whether vitamin $D$ deficiency may contribute to the metabolic disturbances commonly found in PCOS women.

\section{REFERENCES}

1. March WA, Moore VM, Willsen KJ et al. The prevalence of polycystic ovary syndrome in a community sample assessed under contrasting diagnostic criteria. Hum Reprod. 2010; 25(2):544-51. 2. Alvarez-Blasco F, Botella-Carretero JI, San Millan $\mathrm{JL}$ et al. Prevalence and characteristics of the polycystic ovary syndrome in overweight and obese women. Arch Intern Med. 2006; 166(19):2081-6.

3. Mahmoudi T. Genetic variation in the vitamin $D$ receptor and polycystic ovary syndrome risk. Fertil Steril. 2009; 92(4):1381-3. 